\title{
EXISTENCE AND UNIQUENESS OF SOLUTIONS FOR NONLINEAR IMPLICIT HADAMARD FRACTIONAL DIFFERENTIAL EQUATIONS WITH NONLOCAL CONDITIONS IN A WEIGHTED BANACH SPACE
}

\author{
ABDELOUAHEB ARDJOUNI ${ }^{1, *}$, ADEL LACHOURI ${ }^{2}$ \\ ${ }^{1}$ Department of Mathematics and Informatics, University of Souk Ahras, Algeria \\ ${ }^{2}$ Applied Mathematics Lab, Department of Mathematics, University of Annaba, Algeria \\ *Corresponding author: abd_ardjouni@yahoo.fr \\ Received Sep. 20, 2021
}

\begin{abstract}
Авstract. The aim of this paper is to prove the existence and uniqueness of solutions for a nonlinear implicit Hadamard fractional differential equation with nonlocal conditions in a weighted Banach space. Our results are based on the Banach and Krasnoselskii fixed point theorems. An example is given to illustrate our obtained results.

2010 Mathematics Subject Classification. 26A33; 34A08.
\end{abstract}

Key words and phrases. fractional differential equations; Hadamard fractional derivatives; fixed point theorems; existence; uniqueness.

\section{INTRODUCTION}

Fractional differential equations arise from a variety of applications including in various fields of science and engineering. In particular, problems concerning qualitative analysis of fractional differential equations have received the attention of many authors, see [1]- [25], [27][31] and the references therein.

Fractional differential equations involving Riemann-Liouville and Caputo fractional derivatives have been studied extensively by several researchers. However, the literature on Hadamard differential equations is not yet as enriched. The fractional derivative due to Hadamard differs from the aforementioned derivatives in the sense that the kernel of the integral in the definition of Hadamard fractional derivative contains a logarithmic function of arbitrary exponent, see $[4-6,10,16]$.

DOI: 10.28924/APJM/8-20 
In [20], Lachouri et al. discussed the existence and uniqueness of solutions of the following implicit fractional differential equation with nonlocal conditions

$$
\left\{\begin{array}{l}
D_{0^{+}}^{\alpha} x(t)=f\left(t, x(t), D_{0^{+}}^{\alpha} x(t)\right), t \in(0, T] \\
\left.t^{1-\alpha} x(t)\right|_{t=0}=x_{0}-g(x), x_{0} \in \mathbb{R}
\end{array}\right.
$$

where $D_{0^{+}}^{\alpha}$ is the standard Riemann-Liouville fractional derivative of order $0<\alpha<1, f$ : $(0, T] \times \mathbb{R} \times \mathbb{R} \rightarrow \mathbb{R}$ and $g: C((0, T], \mathbb{R}) \rightarrow \mathbb{R}$ are continuous functions. By employing the Banach and Krasnoselskii fixed point theorems, the authors obtained existence and uniqueness results.

In this paper, we extend the results in [20] by proving the existence and uniqueness of solutions for the following implicit Hadamard fractional differential equation with nonlocal conditions in a weighted Banach space

$$
\left\{\begin{array}{l}
D_{1^{+}}^{\alpha} x(t)=f\left(t, x(t), D_{1^{+}}^{\alpha} x(t)\right), t \in(1, T] \\
\left.(\log t)^{1-\alpha} x(t)\right|_{t=1}=x_{0}-g(x), x_{0} \in \mathbb{R}
\end{array}\right.
$$

where $D_{1^{+}}^{\alpha}$ is the Hadamard fractional derivative of order $0<\alpha<1, f:(1, T] \times \mathbb{R} \times \mathbb{R} \rightarrow \mathbb{R}$ and $g: C((1, T], \mathbb{R}) \rightarrow \mathbb{R}$ are continuous nonlinear functions. To prove the existence and uniqueness of solutions in a weighted Banach space, we transform (1) into an equivalent integral equation and then use the Banach and Krasnoselskii fixed point theorems. Finally, we provide an example to illustrate our obtained results.

\section{Preliminaries}

Let $T>1, J=[1, T]$. By $C(J, \mathbb{R})$ we denote the Banach space of all continuous functions from $J$ into $\mathbb{R}$ with the norm

$$
\|x\|_{\infty}=\sup \{|x(t)|: t \in J\}
$$

In what follows $\gamma>0$, we consider the weighted space of continuous functions

$$
C_{\gamma}(J, \mathbb{R})=\left\{x:(1, T] \rightarrow \mathbb{R}:(\log t)^{\gamma} x \in C(J, \mathbb{R})\right\}
$$

with the norm

$$
\|x\|_{C_{\gamma}}=\sup _{t \in J}\left|(\log t)^{\gamma} x(t)\right| .
$$

Clearly $C_{\gamma}(J, \mathbb{R})$ is a Banach space. 
Definition 1 ( [16]). The Hadamard fractional integral of order $\alpha>0$ of a function $x: J \rightarrow \mathbb{R}$ is given by

$$
I_{1^{+}}^{\alpha} x(t)=\frac{1}{\Gamma(\alpha)} \int_{1}^{t}\left(\log \frac{t}{s}\right)^{\alpha-1} x(s) \frac{d s}{s},
$$

where $\Gamma$ is the gamma function.

Definition 2 ( [16]). The Hadamard fractional derivative of order $\alpha>0$ of $x: J \rightarrow \mathbb{R}$ is defined by

$$
D_{1^{+}}^{\alpha} x(t)=\frac{1}{\Gamma(n-\alpha)}\left(t \frac{d}{d t}\right)^{n} \int_{1}^{t}\left(\log \frac{t}{s}\right)^{n-\alpha-1} x(s) \frac{d s}{s}
$$

where $n=[\alpha]+1$ and $[\alpha]$ denotes the integer part of real number $\alpha$.

Lemma 1 ( [16]). The general solution of linear fractional differential equation

$$
D_{1^{+}}^{\alpha} x(t)=0
$$

is given by

$$
x(t)=c_{1}(\log t)^{\alpha-1}+c_{2}(\log t)^{\alpha-2}+c_{3}(\log t)^{\alpha-3}+\ldots+c_{n}(\log t)^{\alpha-n}, c_{i} \in \mathbb{R}, i=1,2, \ldots, n,
$$

where $n=[\alpha]+1$.

Lemma 2 ( [16]). We have

$$
I_{1^{+}}^{\alpha}(\log t)^{\beta-1}=\frac{\Gamma(\beta)}{\Gamma(\alpha+\beta)}(\log t)^{\alpha+\beta-1}, \alpha \geq 0, \beta>0
$$

Theorem 1 (Banach's fixed point theorem [26]). Let $\Omega$ be a non-empty closed convex subset of a Banach space $(S,\|\|$.$) , then any contraction mapping \Phi$ of $\Omega$ into itself has a unique fixed point.

Theorem 2 (Krasnoselskii's fixed point theorem [26]). Let $\Omega$ be a non-empty closed bounded convex subset of a Banach space $(S,\|\|$.$) . Suppose that F_{1}$ and $F_{2}$ map $\Omega$ into $S$ such that

(i) $F_{1} x+F_{2} y \in \Omega$ for all $x, y \in \Omega$,

(ii) $F_{1}$ is continuous and compact,

(iii) $F_{2}$ is a contraction with constant $l<1$.

Then there is $a z \in \Omega$ with $F_{1} z+F_{2} z=z$. 


\section{EXISTENCE AND UNIQUENESS}

Definition 3. A function $x \in C^{1}((1, T], \mathbb{R})$ is said to be a solution of $(1)$ if $x$ satisfies $D_{1^{+}}^{\alpha} x(t)=$ $f\left(t, x(t), D_{1^{+}}^{\alpha} x(t)\right)$ for any $t \in(1, T]$ and $\left.(\log t)^{1-\alpha} x(t)\right|_{t=1}=x_{0}-g(x)$.

For the existence of solutions for the problem (1), we need the following auxiliary lemma.

Lemma 3. The function $x$ solves (1) if and only if it is a solution of the integral equation

(2) $x(t)=(\log t)^{\alpha-1}\left(x_{0}-g(x)\right)+\frac{1}{\Gamma(\alpha)} \int_{1}^{t}\left(\log \frac{t}{s}\right)^{\alpha-1} f\left(s, x(s), D_{1^{+}}^{\alpha} x(s)\right) \frac{d s}{s}, t \in(1, T]$.

Proof. Suppose the function $x$ satisfies (1), then applying $I_{1^{+}}^{\alpha}$ to both sides of $(1)$, we get

$$
I_{1^{+}}^{\alpha} D_{1^{+}}^{\alpha} x(t)=I_{1^{+}}^{\alpha} f\left(t, x(t), D_{1^{+}}^{\alpha} x(t)\right)
$$

By using Lemma 1, we obtain

$$
x(t)=c_{1}(\log t)^{\alpha-1}+\frac{1}{\Gamma(\alpha)} \int_{1}^{t}\left(\log \frac{t}{s}\right)^{\alpha-1} f\left(s, x(s), D_{1^{+}}^{\alpha} x(s)\right) \frac{d s}{s} .
$$

The condition $\left.(\log t)^{1-\alpha} x(t)\right|_{t=1}=x_{0}-g(x)$ implies that

$$
c_{1}=x_{0}-g(x)
$$

Substituting (4) in (3) we get the integral equation (2). The converse can be proven by direct computations. The proof is completed.

In the following subsections we prove existence, as well as existence and uniqueness results, for the problem (1) by using a variety of fixed point theorems.

The following assumptions will be used in our main results.

(H1) There exist constants $k_{1}>0$ and $k_{2} \in(0,1)$ such that

$$
\left|f(t, u, v)-f\left(t, u^{*}, v^{*}\right)\right| \leq k_{1}\left|u-u^{*}\right|+k_{2}\left|v-v^{*}\right|
$$

for $t \in(1, T], u, v, u^{*}, v^{*} \in \mathbb{R}$ and $f(., 0,0) \in C_{1-\alpha}(J, \mathbb{R})$.

(H2) There exist a constant $b \in(0,1)$ such that

$$
\left|g(u)-g\left(u^{*}\right)\right| \leq b\left\|u-u^{*}\right\|_{C_{1-\alpha}}
$$

for $u, u^{*} \in C_{1-\alpha}(J, \mathbb{R})$. 


\subsection{Existence and uniqueness results via Banach's fixed point theorem.}

Theorem 3. Suppose that (H1) and (H2) hold. If

$$
b+\frac{\Gamma(\alpha) k_{1}(\log T)^{\alpha}}{\Gamma(2 \alpha)\left(1-k_{2}\right)}<1
$$

then there exists a unique solution for $(1)$ in the space $C_{1-\alpha}(J, \mathbb{R})$.

Proof. We define the operator $\Phi: C_{1-\alpha}(J, \mathbb{R}) \rightarrow C_{1-\alpha}(J, \mathbb{R})$ by

$$
(\Phi x)(t)=(\log t)^{\alpha-1}\left(x_{0}-g(x)\right)+\frac{1}{\Gamma(\alpha)} \int_{1}^{t}\left(\log \frac{t}{s}\right)^{\alpha-1} h(s) \frac{d s}{s}, t \in(1, T],
$$

where $h:(1, T] \rightarrow \mathbb{R}$ be a function satisfying the functional equation

$$
h(t)=f(t, x(t), h(t)) .
$$

By Lemma 3, the fixed points of operator $\Phi$ are solutions of (1). The operator $\Phi$ is well define, i.e. for every $x \in C_{1-\alpha}(J, \mathbb{R})$ and $t>1$, the integral

$$
\frac{1}{\Gamma(\alpha)} \int_{1}^{t}\left(\log \frac{t}{s}\right)^{\alpha-1} h(s) \frac{d s}{s}
$$

belongs to $C_{1-\alpha}(J, \mathbb{R})$. Under the condition $(\mathrm{H} 1)$, we get

$$
\begin{aligned}
|h(t)| & =|f(t, x(t), h(t))| \\
& \leq \frac{k_{1}}{1-k_{2}}|x(t)|+c(\log t)^{\alpha-1} \text { for each } t \in(1, T],
\end{aligned}
$$

where $c=\frac{\sup _{t \in J}\left|(\log t)^{1-\alpha} f(t, 0,0)\right|}{1-k_{2}}$. For every $x \in C_{1-\alpha}(J, \mathbb{R})$, we obtain

$$
\begin{aligned}
& \left|\frac{(\log t)^{1-\alpha}}{\Gamma(\alpha)} \int_{1}^{t}\left(\log \frac{t}{s}\right)^{\alpha-1} h(s) \frac{d s}{s}\right| \\
& \leq \frac{(\log t)^{1-\alpha}}{\Gamma(\alpha)} \int_{1}^{t}\left(\log \frac{t}{s}\right)^{\alpha-1}|h(s)| \frac{d s}{s} \\
& \leq \frac{(\log t)^{1-\alpha}}{\Gamma(\alpha)} \int_{1}^{t}\left(\log \frac{t}{s}\right)^{\alpha-1}\left(\frac{k_{1}}{1-k_{2}}|x(s)|+c(\log s)^{\alpha-1}\right) \frac{d s}{s} \\
& \leq \frac{(\log t)^{1-\alpha}}{\Gamma(\alpha)} \int_{1}^{t}\left(\log \frac{t}{s}\right)^{\alpha-1}(\log s)^{\alpha-1}\left(\frac{k_{1}}{1-k_{2}}\left|(\log s)^{1-\alpha} x(s)\right|+c\right) \frac{d s}{s} \\
& \leq\left(\frac{k_{1}}{1-k_{2}}\|x\|_{C_{1-\alpha}}+c\right)(\log t)^{1-\alpha} I_{1^{+}}^{\alpha}\left((\log t)^{\alpha-1}\right) .
\end{aligned}
$$


By Lemma 2, we have

$$
\begin{aligned}
& \left|\frac{(\log t)^{1-\alpha}}{\Gamma(\alpha)} \int_{1}^{t}\left(\log \frac{t}{s}\right)^{\alpha-1} h(s) \frac{d s}{s}\right| \\
& \leq\left(\frac{k_{1}}{1-k_{2}}\|x\|_{C_{1-\alpha}}+c\right) \frac{\Gamma(\alpha)(\log t)^{\alpha}}{\Gamma(2 \alpha)} \\
& \leq\left(\frac{k_{1}}{1-k_{2}}\|x\|_{C_{1-\alpha}}+c\right) \frac{\Gamma(\alpha)(\log T)^{\alpha}}{\Gamma(2 \alpha)} .
\end{aligned}
$$

That is to say that the integral exists and belongs to $C_{1-\alpha}(J, \mathbb{R})$.

Let $x, y \in C_{1-\alpha}(J, \mathbb{R})$. Then for $t \in(1, T]$, we obtain

$$
\begin{aligned}
& |(\Phi x)(t)-(\Phi y)(t)| \\
& \leq(\log t)^{\alpha-1}|g(x)-g(y)|+\frac{1}{\Gamma(\alpha)} \int_{1}^{t}\left(\log \frac{t}{s}\right)^{\alpha-1}\left|h_{x}(s)-h_{y}(s)\right| \frac{d s}{s}
\end{aligned}
$$

where $h_{x}, h_{y} \in C_{1-\alpha}(J, \mathbb{R})$ be such that

$$
h_{x}(t)=f\left(t, x(t), h_{x}(t)\right)
$$

and

$$
h_{y}(t)=f\left(t, y(t), h_{y}(t)\right)
$$

By $(\mathrm{H} 1)$ we get

$$
\begin{aligned}
\left|h_{x}(t)-h_{y}(t)\right| & =\left|f\left(t, x(t), h_{x}(t)\right)-f\left(t, y(t), h_{y}(t)\right)\right| \\
& \leq k_{1}|x(t)-y(t)|+k_{2}\left|h_{x}(t)-h_{y}(t)\right|
\end{aligned}
$$

Then

$$
\left|h_{x}(t)-h_{y}(t)\right| \leq \frac{k_{1}}{1-k_{2}}|x(t)-y(t)| .
$$

Therefore, for each $t \in(1, T]$, we have

$$
\begin{aligned}
& |(\Phi x)(t)-(\Phi y)(t)| \\
& \leq b(\log t)^{\alpha-1}\|x-y\|_{C_{1-\alpha}}+\frac{k_{1}}{\Gamma(\alpha)\left(1-k_{2}\right)} \int_{1}^{t}\left(\log \frac{t}{s}\right)^{\alpha-1}|x(s)-y(s)| \frac{d s}{s} \\
& =(\log t)^{\alpha-1} b\|x-y\|_{C_{1-\alpha}} \\
& +\frac{k_{1}}{\Gamma(\alpha)\left(1-k_{2}\right)} \int_{1}^{t}\left(\log \frac{t}{s}\right)^{\alpha-1}(\log s)^{\alpha-1}\left|(\log s)^{1-\alpha}(x(s)-y(s))\right| \frac{d s}{s} \\
& \leq(\log t)^{\alpha-1} b\|x-y\|_{C_{1-\alpha}}+\frac{k_{1}}{1-k_{2}} I_{1^{+}}^{\alpha}\left((\log t)^{\alpha-1}\right)\|x-y\|_{C_{1-\alpha}} .
\end{aligned}
$$


By Lemma 2, we get

$$
|(\Phi x)(t)-(\Phi y)(t)| \leq(\log t)^{\alpha-1} b\|x-y\|_{C_{1-\alpha}}+\frac{\Gamma(\alpha) k_{1}(\log t)^{2 \alpha-1}}{\Gamma(2 \alpha)\left(1-k_{2}\right)}\|x-y\|_{C_{1-\alpha}},
$$

which implies that

$$
\begin{aligned}
& \left|(\log t)^{1-\alpha}((\Phi x)(t)-(\Phi y)(t))\right| \\
& \leq b\|x-y\|_{C_{1-\alpha}}+\frac{\Gamma(\alpha) k_{1}(\log t)^{\alpha}}{\Gamma(2 \alpha)\left(1-k_{2}\right)}\|x-y\|_{C_{1-\alpha}} \\
& \leq b\|x-y\|_{C_{1-\alpha}}+\frac{\Gamma(\alpha) k_{1}(\log T)^{\alpha}}{\Gamma(2 \alpha)\left(1-k_{2}\right)}\|x-y\|_{C_{1-\alpha}} .
\end{aligned}
$$

Thus

$$
\|\Phi x-\Phi y\|_{C_{1-\alpha}} \leq\left(b+\frac{\Gamma(\alpha) k_{1}(\log T)^{\alpha}}{\Gamma(2 \alpha)\left(1-k_{2}\right)}\right)\|x-y\|_{C_{1-\alpha}} .
$$

From (5), $\Phi$ is a contraction. As a consequence of Banach's fixed point theorem, we get that $\Phi$ has a unique fixed point which is a unique solution of the problem (1).

\subsection{Existence results via Krasnoselskii's fixed point theorem.}

Theorem 4. Suppose that (H1), (H2) hold and the following hypothesis

(H3) There exist $p_{1} \in C_{1-\alpha}\left(J, \mathbb{R}^{+}\right), p_{2}, p_{3} \in C\left(J, \mathbb{R}^{+}\right)$with $p_{3}^{*}=\sup _{t \in J} p_{3}(t)<1$ such that

$$
|f(t, u, v)| \leq p_{1}(t)+p_{2}(t)|u|+p_{3}(t)|v|,
$$

for $t \in(1, T]$ and each $u, v \in \mathbb{R}$.

If

$$
\lambda=b+\frac{p_{2}^{*} \Gamma(\alpha)(\log T)^{\alpha}}{\left(1-p_{3}^{*}\right) \Gamma(2 \alpha)}<1,
$$

where $p_{2}^{*}=\sup _{t \in J} p_{2}(t)$. Then (1) has at least one solution in $\Omega$.

Proof. Set

$$
R=\frac{1}{1-\lambda}, \Lambda=\left|x_{0}\right|+Q+\frac{(\log T)^{\alpha} p_{1}^{*} \Gamma(\alpha)}{\left(1-p_{3}^{*}\right) \Gamma(2 \alpha)},
$$

where $p_{1}^{*}=\sup _{t \in J}\left\{(\log t)^{1-\alpha} p_{1}(t)\right\}$ and $Q=|g(0)|$. Let us fix

$$
M \geq R \Lambda .
$$

Consider the non-empty closed bounded convex subset $\Omega=\left\{x \in C_{1-\alpha}(J, \mathbb{R}):\|x\|_{C_{1-\alpha}} \leq M\right\}$ and define two operators $F_{1}$ and $F_{2}$ on $\Omega$, as follows

$$
\left(F_{1} x\right)(t)=\frac{1}{\Gamma(\alpha)} \int_{1}^{t}\left(\log \frac{t}{s}\right)^{\alpha-1} h(s) \frac{d s}{s},
$$


and

$$
\left(F_{2} x\right)(t)=(\log t)^{\alpha-1}\left(x_{0}-g(x)\right),
$$

where $h:(1, T] \rightarrow \mathbb{R}$ be a function satisfying the functional equation

$$
h(t)=f(t, x(t), h(t)) .
$$

We shall use the Krasnoselskii fixed point theorem to show there exists at least one fixed point of the operator $F_{1}+F_{2}$ in $\Omega$. The proof will be given in several steps.

Step 1. We prove that $F_{1} x+F_{2} y \in \Omega$ for all $x, y \in \Omega$.

For any $x, y \in \Omega$ and $t \in(1, T]$, we have

$$
\begin{aligned}
& \left|\left(F_{1} x\right)(t)+\left(F_{2} y\right)(t)\right| \\
& \leq\left|(\log t)^{\alpha-1}\left(x_{0}-g(x)\right)+\frac{1}{\Gamma(\alpha)} \int_{1}^{t}\left(\log \frac{t}{s}\right)^{\alpha-1} h(s) \frac{d s}{s}\right| \\
& \leq(\log t)^{\alpha-1}\left|x_{0}\right|+(\log t)^{\alpha-1}|g(x)-g(0)|+(\log t)^{\alpha-1}|g(0)| \\
& +\frac{1}{\Gamma(\alpha)} \int_{1}^{t}\left(\log \frac{t}{s}\right)^{\alpha-1}(\log s)^{\alpha-1}\left|(\log s)^{1-\alpha} h(s)\right| \frac{d s}{s} \\
& \leq(\log t)^{\alpha-1}\left|x_{0}\right|+(\log t)^{\alpha-1} b\|x\|_{C_{1-\alpha}}+(\log t)^{\alpha-1} Q \\
& +\frac{1}{\Gamma(\alpha)} \int_{1}^{t}\left(\log \frac{t}{s}\right)^{\alpha-1}(\log s)^{\alpha-1}\left|(\log s)^{1-\alpha} h(s)\right| \frac{d s}{s} \\
& \leq(\log t)^{\alpha-1}\left|x_{0}\right|+(\log t)^{\alpha-1} b M+(\log t)^{\alpha-1} Q \\
& +\frac{1}{\Gamma(\alpha)} \int_{1}^{t}\left(\log \frac{t}{s}\right)^{\alpha-1}(\log s)^{\alpha-1}\left|(\log s)^{1-\alpha} h(s)\right| \frac{d s}{s} .
\end{aligned}
$$

By (H3), for each $t \in(1, T]$, we obtain

$$
\begin{aligned}
|h(t)| & =|f(t, x(t), h(t))| \\
& \leq p_{1}(t)+p_{2}(t)|x(t)|+p_{3}(t)|h(t)|
\end{aligned}
$$

Hence, we get

$$
\begin{aligned}
\left|(\log t)^{1-\alpha} h(t)\right| & \leq(\log t)^{1-\alpha} p_{1}(t)+p_{2}(t)\left|(\log t)^{1-\alpha} x(t)\right|+p_{3}(t)\left|(\log t)^{1-\alpha} h(t)\right| \\
& \leq p_{1}^{*}+p_{2}^{*} M+p_{3}^{*}\left|(\log t)^{1-\alpha} h(t)\right|
\end{aligned}
$$

then, we have

$$
\left|(\log t)^{1-\alpha} h(t)\right| \leq \frac{p_{1}^{*}+p_{2}^{*} M}{1-p_{3}^{*}}
$$


Replacing (9) in the inequality (8) and with Lemma 2, we obtain

$$
\begin{aligned}
& \left|\left(F_{1} x\right)(t)+\left(F_{2} y\right)(t)\right| \\
& \leq(\log t)^{\alpha-1}\left|x_{0}\right|+(\log t)^{\alpha-1} b M+(\log t)^{\alpha-1} Q \\
& +\left(\frac{p_{1}^{*}+p_{2}^{*} M}{1-p_{3}^{*}}\right) \frac{1}{\Gamma(\alpha)} \int_{1}^{t}\left(\log \frac{t}{s}\right)^{\alpha-1}(\log s)^{\alpha-1} \frac{d s}{s} \\
& \leq(\log t)^{\alpha-1}\left|x_{0}\right|+(\log t)^{\alpha-1} b M+(\log t)^{\alpha-1} Q+\left(\frac{p_{1}^{*}+p_{2}^{*} M}{1-p_{3}^{*}}\right) \frac{\Gamma(\alpha)}{\Gamma(2 \alpha)}(\log t)^{2 \alpha-1} .
\end{aligned}
$$

Therefore

$$
\begin{aligned}
& \left|(\log t)^{1-\alpha}\left(\left(F_{1} x\right)(t)+\left(F_{2} x\right)(t)\right)\right| \\
& \leq\left|x_{0}\right|+Q+\frac{(\log T)^{\alpha} p_{1}^{*} \Gamma(\alpha)}{\left(1-p_{3}^{*}\right) \Gamma(2 \alpha)}+\left(b+\frac{p_{2}^{*} \Gamma(\alpha)(\log T)^{\alpha}}{\left(1-p_{3}^{*}\right) \Gamma(2 \alpha)}\right) M .
\end{aligned}
$$

Thus

$$
\begin{aligned}
& \left\|F_{1} x+F_{2} x\right\|_{C_{1-\alpha}} \\
& \leq \Lambda+\lambda M \leq \frac{M}{R}+\left(1-\frac{1}{R}\right) M=M .
\end{aligned}
$$

Hence $F_{1} x+F_{2} y \in \Omega$ for all $x, y \in \Omega$.

Step 2. We show that $F_{1}$ is continuous.

Let $\left(x_{n}\right)_{n \in \mathbb{N}}$ be a sequence such that $x_{n} \rightarrow x$ in $C_{1-\alpha}(J, \mathbb{R})$, then for each $t \in(1, T]$, we have

$$
\left|\left(F_{1} x_{n}\right)(t)-\left(F_{1} x\right)(t)\right| \leq \frac{1}{\Gamma(\alpha)} \int_{1}^{t}\left(\log \frac{t}{s}\right)^{\alpha-1}\left|h_{n}(s)-h(s)\right| \frac{d s}{s},
$$

where $h_{n}, h \in C_{1-\alpha}(J, \mathbb{R})$ be such that

$$
h_{n}(t)=f\left(t, x_{n}(t), h_{n}(t)\right),
$$

and

$$
h(t)=f(t, x(t), h(t)) .
$$

By (H1) we have

$$
\begin{aligned}
\left|h_{n}(t)-h(t)\right| & =\left|f\left(t, x_{n}(t), h_{n}(t)\right)-f(t, x(t), h(t))\right| \\
& \leq k_{1}\left|x_{n}(t)-x(t)\right|+k_{2}\left|h_{n}(t)-h(t)\right|
\end{aligned}
$$

Then

$$
\left|h_{n}(t)-h(t)\right| \leq \frac{k_{1}}{1-k_{2}}\left|x_{n}(t)-x(t)\right|
$$


By replacing (11) in inequality (10), we get

$$
\begin{aligned}
& \left|\left(F_{1} x_{n}\right)(t)-\left(F_{1} x\right)(t)\right| \\
& \leq \frac{k_{1}}{\left(1-k_{2}\right) \Gamma(\alpha)} \int_{1}^{t}\left(\log \frac{t}{s}\right)^{\alpha-1}\left|x_{n}(t)-x(t)\right| \frac{d s}{s} \\
& =\frac{k_{1}}{\left(1-k_{2}\right) \Gamma(\alpha)} \int_{1}^{t}\left(\log \frac{t}{s}\right)^{\alpha-1}(\log s)^{\alpha-1}\left|(\log s)^{1-\alpha}\left(x_{n}(t)-x(t)\right)\right| \frac{d s}{s} \\
& \leq \frac{k_{1}}{1-k_{2}} I_{1^{+}}^{\alpha}\left((\log t)^{\alpha-1}\right)\left\|x_{n}-x\right\|_{C_{1-\alpha}} .
\end{aligned}
$$

By Lemma 2, we obtain

$$
\left|\left(F_{1} x_{n}\right)(t)-\left(F_{1} x\right)(t)\right| \leq \frac{\Gamma(\alpha) k_{1}(\log t)^{2 \alpha-1}}{\left(1-k_{2}\right) \Gamma(2 \alpha)}\left\|x_{n}-x\right\|_{C_{1-\alpha}},
$$

which implies that

$$
\begin{aligned}
\left|(\log t)^{1-\alpha}\left(\left(F_{1} x_{n}\right)(t)-\left(F_{1} x\right)(t)\right)\right| & \leq \frac{\Gamma(\alpha) k_{1}(\log t)^{\alpha}}{\left(1-k_{2}\right) \Gamma(2 \alpha)}\left\|x_{n}-x\right\|_{C_{1-\alpha}} \\
& \leq \frac{\Gamma(\alpha) k_{1}(\log T)^{\alpha}}{\left(1-k_{2}\right) \Gamma(2 \alpha)}\left\|x_{n}-x\right\|_{C_{1-\alpha}} .
\end{aligned}
$$

Thus

$$
\left\|F_{1} x_{n}-F_{1} x\right\|_{C_{1-\alpha}} \leq \frac{\Gamma(\alpha) k_{1}(\log T)^{\alpha}}{\left(1-k_{2}\right) \Gamma(2 \alpha)}\left\|x_{n}-x\right\|_{C_{1-\alpha}}
$$

and hence

$$
\left\|F_{1} x_{n}-F_{1} x\right\|_{C_{1-\alpha}} \rightarrow 0 \text { as } n \rightarrow \infty .
$$

Consequently, $F_{1}$ is continuous.

Step 3. We prove that $F_{1}$ is compact.

For all $x \in \Omega$ and $t \in(1, T]$, we have

$$
\left|\left(F_{1} x\right)(t)\right| \leq \frac{1}{\Gamma(\alpha)} \int_{1}^{t}\left(\log \frac{t}{s}\right)^{\alpha-1}(\log s)^{\alpha-1}\left|(\log s)^{1-\alpha} h(s)\right| \frac{d s}{s} .
$$

Replacing (9) in the inequality (12) and with Lemma 2, we obtain

$$
\left|\left(F_{1} x\right)(t)\right| \leq\left(\frac{p_{1}^{*}+p_{2}^{*} M}{1-p_{3}^{*}}\right) \frac{\Gamma(\alpha)}{\Gamma(2 \alpha)}(\log t)^{2 \alpha-1} .
$$

Therefore

$$
\left|(\log t)^{1-\alpha}\left(F_{1} x\right)(t)\right| \leq\left(\frac{p_{1}^{*}+p_{2}^{*} M}{1-p_{3}^{*}}\right) \frac{\Gamma(\alpha)}{\Gamma(2 \alpha)}(\log T)^{\alpha}
$$

Thus

$$
\left\|F_{1} x\right\|_{C_{1-\alpha}} \leq\left(\frac{p_{1}^{*}+p_{2}^{*} M}{1-p_{3}^{*}}\right) \frac{\Gamma(\alpha)}{\Gamma(2 \alpha)}(\log T)^{\alpha} .
$$

Hence $F_{1}(\Omega)$ is uniformly bounded. 
It remains to show that $F_{1}(\Omega)$ is equicontinuous, let $1 \leq t_{1}<t_{2} \leq T$ and $x \in \Omega$. Then

$$
\begin{aligned}
& \left|\left(\log t_{2}\right)^{1-\alpha}\left(F_{1} x\right)\left(t_{2}\right)-\left(\log t_{1}\right)^{1-\alpha}\left(F_{1} x\right)\left(t_{1}\right)\right| \\
& =\frac{1}{\Gamma(\alpha)} \mid \int_{1}^{t_{1}}\left(\log t_{2}\right)^{1-\alpha}\left(\log \frac{t_{2}}{s}\right)^{\alpha-1} h(s) \frac{d s}{s}+\int_{t_{1}}^{t_{2}}\left(\log t_{2}\right)^{1-\alpha}\left(\log \frac{t_{2}}{s}\right)^{\alpha-1} h(s) \frac{d s}{s} \\
& -\int_{1}^{t_{1}}\left(\log t_{1}\right)^{1-\alpha}\left(\log \frac{t_{1}}{s}\right)^{\alpha-1} h(s) \frac{d s}{s} \\
& \leq \frac{1}{\Gamma(\alpha)} \int_{1}^{t_{1}} \mid\left(\log t_{2}\right)^{1-\alpha}\left(\log \frac{t_{2}}{s}\right)^{\alpha-1}(\log s)^{\alpha-1} \\
& -\left(\log t_{1}\right)^{1-\alpha}\left(\log \frac{t_{1}}{s}\right)^{\alpha-1}(\log s)^{\alpha-1}||(\log s)^{1-\alpha} h(s) \mid \frac{d s}{s} \\
& +\frac{1}{\Gamma(\alpha)} \int_{t_{1}}^{t_{2}}\left(\log t_{2}\right)^{1-\alpha}\left(\log \frac{t_{2}}{s}\right)^{\alpha-1}(\log s)^{\alpha-1}\left|(\log s)^{1-\alpha} h(s)\right| \frac{d s}{s} \\
& \leq \frac{p_{1}^{*}+p_{2}^{*} M}{1-p_{3}^{*}}\left(\frac{1}{\Gamma(\alpha)} \int_{1}^{t_{1}} \mid\left(\log t_{2}\right)^{1-\alpha}\left(\log \frac{t_{2}}{s}\right)^{\alpha-1}\right. \\
& \left.-\left(\log t_{1}\right)^{1-\alpha}\left(\log \frac{t_{1}}{s}\right)^{\alpha-1} \mid(\log s)^{\alpha-1} \frac{d s}{s}\right) \\
& +\frac{p_{1}^{*}+p_{2}^{*} M}{1-p_{3}^{*}}\left(\frac{1}{\Gamma(\alpha)} \int_{t_{1}}^{t_{2}}\left(\log t_{2}\right)^{1-\alpha}\left(\log \frac{t_{2}}{s}\right)^{\alpha-1}(\log s)^{\alpha-1} \frac{d s}{s}\right) .
\end{aligned}
$$

As $t_{1} \rightarrow t_{2}$, the right-hand side of the above inequality tends to zero. That is to say that $F_{1}(\Omega)$ is equicontinuous, then by Ascoli-Arzela theorem, we can conclude that the operator $F_{1}$ is compact.

Step 4. We prove that $F_{2}: \Omega \rightarrow C_{1-\alpha}(J, \mathbb{R})$ is a contraction mapping.

For all $x \in \Omega$ and from (H2), we get

$$
\begin{aligned}
\left|\left(F_{2} x\right)(t)-\left(F_{2} y\right)(t)\right| & =\left|(\log t)^{\alpha-1}(g(x)-g(y))\right| \\
& \leq(\log t)^{\alpha-1} b\|x-y\|_{C_{1-\alpha}}
\end{aligned}
$$

Therefore

$$
\left|(\log t)^{1-\alpha}\left(\left(F_{2} x\right)(t)-\left(F_{2} y\right)(t)\right)\right| \leq b\|x-y\|_{C_{1-\alpha}} .
$$

Then

$$
\left\|F_{2} x-F_{2} y\right\|_{C_{1-\alpha}} \leq b\|x-y\|_{C_{1-\alpha}} .
$$

Hence, the operator $F_{2}$ is a contraction. 
Clearly, all the hypotheses of the Krasnoselskii fixed point theorem (see [26]) are satisfied. Thus there a fixed point $x \in \Omega$ such that $x=F_{1} x+F_{2} x$, which is a solution of the problem (1).

\section{EXAMPLE}

We consider the following fractional initial value problem

$$
\left\{\begin{array}{l}
D_{1^{+}}^{\frac{3}{4}} x(t)=\frac{1}{5 \exp (-t+3)\left(2+|x(t)|+\left|D_{1^{+}}^{\frac{3}{4}} x(t)\right|\right)}+\frac{1}{(\log t)^{\frac{1}{4}}}, t \in(1, e] \\
\left.(\log t)^{\frac{1}{4}} x(t)\right|_{t=0}=\frac{1}{2}-\sum_{i=1}^{n} c_{i}\left(\log t_{i}\right)^{\frac{1}{4}} x\left(t_{i}\right)
\end{array}\right.
$$

where $1<t_{1}<\ldots<t_{n}<e$ and $c_{i}, i=1, \ldots, n$ are positive constants with

$$
\sum_{i=1}^{n} c_{i} \leq \frac{1}{3}
$$

Set

$$
f(t, u, v)=\frac{1}{5 \exp (-t+3)(2+|u|+|v|)}+\frac{1}{(\log t)^{\frac{1}{4}}}, t \in(1, e], u, v \in \mathbb{R}
$$

We have

$$
C_{1-\alpha}([1, e], \mathbb{R})=C_{\frac{1}{4}}([1, e], \mathbb{R})=\left\{h:(1, e] \rightarrow \mathbb{R}:(\log t)^{\frac{1}{4}} h \in C([1, e], \mathbb{R})\right\}
$$

with $\alpha=\frac{3}{4}$. Clearly the functions $f$ and $g$ are continuous, $f(., 0,0) \in C_{\frac{1}{4}}([1, e], \mathbb{R})$. For each $u, u^{*}, v, v^{*} \in \mathbb{R}$ and $t \in(1, e]$, we have

$$
\begin{aligned}
& \left|f(t, u, v)-f\left(t, u^{*}, v^{*}\right)\right| \\
& =\left|\frac{1}{5 \exp (-t+3)}\left(\frac{1}{2+|u|+|v|}-\frac{1}{2+\left|u^{*}\right|+\left|v^{*}\right|}\right)\right| \\
& \leq \frac{\left|u-u^{*}\right|+\left|v-v^{*}\right|}{5 \exp (-t+3)(2+|u|+|v|)\left(2+\left|u^{*}\right|+\left|v^{*}\right|\right)} \\
& \leq \frac{1}{20 e^{-e+3}}\left(\left|u-u^{*}\right|+\left|v-v^{*}\right|\right)
\end{aligned}
$$

and

$$
\begin{aligned}
\left|g(u)-g\left(u^{*}\right)\right| & \leq \sum_{i=1}^{n} c_{i}\left(\log t_{i}\right)^{\frac{1}{4}}\left|u\left(t_{i}\right)-u^{*}\left(t_{i}\right)\right| \\
& \leq \sum_{i=1}^{n} c_{i}\left\|u-u^{*}\right\|_{C_{\frac{1}{4}}} \leq \frac{1}{3}\left\|u-u^{*}\right\|_{C_{\frac{1}{4}}}
\end{aligned}
$$


Hence, conditions (H1) and (H2) are satisfied with $k_{1}=k_{2}=\frac{1}{20 e^{-e+3}}$ and $b=\frac{1}{3}$. The condition

$$
b+\frac{\Gamma(\alpha) k_{1}(\log T)^{\alpha}}{\Gamma(2 \alpha)\left(1-k_{2}\right)}=\frac{1}{3}+\frac{\frac{\Gamma\left(\frac{3}{4}\right)}{20 e^{-e+3}}}{\Gamma\left(\frac{3}{2}\right)\left(1-\frac{1}{20 e^{-e+3}}\right)} \simeq 0.388<1,
$$

is satisfied with $T=e$. It follows from Theorem 3 that the problem (13) has a unique solution in the space $C_{\frac{1}{4}}([1, e], \mathbb{R})$.

\section{REFERENCES}

[1] S. Abbas, Existence of solutions to fractional order ordinary and delay differential equations and applications, Electron. J. Differ. Equations 2011 (2011), 09.

[2] R. P. Agarwal, Y. Zhou, Y. He, Existence of fractional functional differential equations, Computers Math. Appl. 59 (2010), 1095-1100.

[3] B. Ahmad, S. K. Ntouyas, Initial-value problems for fractional differential equations, Electron. J. Differ. Equations 2014 (2014), 161.

[4] A. Ardjouni, Positive solutions for nonlinear Hadamard fractional differential equations with integral boundary conditions, AIMS Math. 4(4) (2019), 1101-1113.

[5] A. Ardjouni, A. Djoudi, Approximating solutions of nonlinear hybrid Caputo fractional integro-differential equations via Dhage iteration principle, Ural Math. J. 5(1) (2019), 3-12.

[6] A. Ardjouni, A. Djoudi, Positive solutions for first-order nonlinear Caputo-Hadamard fractional relaxation differential equations, Kragujevac J. Math. 45(6) (2021), 897-908.

[7] Z. Bai, S. Zhang, S.Sun, C. Yin, Monotone iterative method for fractional differential equations, Electron. J. Differ. Equations 2016 (2016), 06.

[8] M. Benchohra, S. Bouriah, M. A. Darwish, Nonlinear boundary value problem for implicit differential equations of fractional order in Banach spaces, Fixed Point Theory 18 (2017), 457-470.

[9] M. Benchohra, S. Bouriah, J. J. Nieto, Existence and Ulam stability for nonlinear implicit differential equations with Riemann-Liouville fractional derivative, Demonstr. Math. 52(1) (2019), 437-450.

[10] M. Benchohra, S. Bouriah, J.J. Nieto, Existence of periodic solutions for nonlinear implicit Hadamard's fractional differential equations, Rev. R. Acad. Cienc. Exactas Fís. Nat., Ser. A Mat. 112 (2018), 25-35.

[11] W. Benhamida, J. R. Graef and S. Hamani, Boundary value problems for fractional differential equations with integral and anti-periodic conditions in a Banach space, Prog. Frac. Differ. Appl. 4(2) (2018), 1-7.

[12] H. Boulares, A. Ardjouni, Y. Laskri, Positive solutions for nonlinear fractional differential equations, Positivity 21 (2017), 1201-1212.

[13] K. Diethelm, The Analysis of Fractional Differential Equations, Lecture Notes in Mathematics, Springerverlag, Berlin, Heidelberg, 2010.

[14] S. Gala, Q. Liu, M.A. Ragusa, A new regularity criterion for the nematic liquid crystal flows, Appl. Anal. 91(9) (2012), 1741-1747.

[15] S. Gala, M.A. Ragusa, Logarithmically improved regularity criterion for the Boussinesq equations in Besov spaces with negative indices, Appl. Anal. 95(6) (2016), 1271-1279. 
[16] A. A. Kilbas, H. M. Srivastava and J. J. Trujillo, Theory and Applications of Fractional Differential Equations, Elsevier Science B. V., Amsterdam, 2006.

[17] C. Kou, H. Zhou, Y. Yan, Existence of solutions of initial value problems for nonlinear fractional differential equations on the half-axis, Nonlinear Anal.: Theory Meth. Appl. 74 (2011), 5975-5986.

[18] K. D. Kucche, J. J. Nieto, V. Venktesh, Theory of nonlinear implicit fractional differential equations, Differ. Equ. Dyn. Syst. 28 (2020), 1-17.

[19] M. Kratou, Ground state solutions of p-Laplacian singular Kirchho problem involving a Riemann-Liouville fractional derivative, Filomat 33(7) (2019), 2073-2088.

[20] A. Lachouri, A. Ardjouni, A. Djoudi, Existence and uniqueness results for nonlinear implicit RiemannLiouville fractional differential equations with nonlocal conditions, Filomat 34(14) (2020), 4881-4891.

[21] V. Lakshmikantham, A. S. Vatsala, Basic theory of fractional differential equations, Nonlinear Anal.: Theory Meth. Appl. 69 (2008), 2677-2682.

[22] D. Luo, Z. Luo, Existence of solutions for fractional differential inclusions with initial value condition and non-instantaneous impulses, Filomat 33(17) (2019), 5499-5510.

[23] J.J. Nieto, A. Ouahab, V. Venktesh, Implicit fractional differential equations via the Liouville-Caputo derivative, Mathematics 3 (2015), 398-411.

[24] I. Podlubny, Fractional Differential Equations, Academic Press, San Diego, 1999.

[25] Samina, K. Shah, R.A. Khan, D. Baleanu, Study of implicit type coupled system of non-integer order differential equations with antiperiodic boundary conditions, Math. Meth. Appl. Sci. 42(6) (2019), 20332042.

[26] D. R. Smart, Fixed Point Theorems, Cambridge Tracts in Mathematics, Cambridge University Press, LondonNew York, 1974.

[27] S. T. Sutar, K. D. Kucche, Global existence and uniqueness for implicit differential equations of arbitrary order, Fract. Differ. Calc. 5(2) (2015), 199-208.

[28] F. Wang, Existence and uniqueness of solutions for a nonlinear fractional differential equation, J. Appl. Math. Comput. 39(1-2) (2012), 53-67.

[29] S. Zhang, The existence of a positive solution for a nonlinear fractional differential equation, J. Math. Anal. Appl. 252 (2000), 804-812.

[30] W. Zhang, W. Liu and T. Xue, Existence and uniqueness results for the coupled systems of implicit fractional differential equations with periodic boundary conditions, Adv. Differ. Equations 2018 (2018), 413.

[31] Y. Zhou, J. R. Wang, L. Zhang, Basic Theory of Fractional Differential Equations, Second edition, World Scientific Publishing Co. Pte. Ltd., Hackensack, NJ, 2017. 